\title{
Repeated Head CT in the Neurosurgical Intensive Care Unit: Feasibility of Sinogram-Affirmed Iterative Reconstruction- Based Ultra-Low-Dose CT for Surveillance
}

\author{
I. Corcuera-Solano, A.H. Doshi, A. Noor, and L.N. Tanenbaum
}

\begin{abstract}
BACKGROUND AND PURPOSE: Patients in the neurosurgical intensive care unit undergo multiple head CT scans, resulting in high cumulative radiation exposures. Our aim was to assess the acceptability of a dedicated, special-purpose sinogram-affirmed iterative reconstruction-based ultra-low-dose CT protocol for neurosurgical intensive care unit surveillance head CT examinations, comparing image quality with studies performed with our standard-of-care sinogram-affirmed iterative reconstruction low-dose CT and legacy filtered back-projection standard-dose CT protocols.
\end{abstract}

MATERIAL AND METHODS: A retrospective analysis was performed of 54 head CT examinations: ultra-low-dose CT ( $n=22)$, low-dose $\mathrm{CT}(n=12)$, and standard-dose CT $(n=20)$ in 22 patients in the neurosurgical intensive care unit. Standard-dose CT was reconstructed by using filtered back-projection on a Somatom Sensation 64 scanner. Ultra-low-dose CT and ultra-low-dose CT examinations were performed on a Siemens AS+128 scanner with commercially available sinogram-affirmed iterative reconstruction. Qualitative and quantitative parameters, including image quality and dose, were evaluated.

RESULTS: Sinogram-affirmed iterative reconstruction ultra-low-dose CT represented a $68 \%$ lower dose index volume compared with filtered back-projection standard-dose CT techniques in the same patients while maintaining similar quality and SNR levels. Sinogramaffirmed iterative reconstruction low-dose CT offered higher image quality than filtered back-projection standard-dose $C T(P<.05)$ with no differences in SNR at a $24 \%$ lower dose index volume. Compared with low-dose CT, ultra-low-dose CT had significantly lower SNR $(P=$ .001) but demonstrated clinically satisfactory measures of image quality.

CONCLUSIONS: In this cohort of patients in the neurosurgical intensive care unit, dedicated ultra-low-dose CT for surveillance head CT imaging led to a significant dose reduction while maintaining adequate image quality.

ABBREVIATIONS: $\mathrm{CTDI}_{\text {vol }}=$ dose index volume; $\mathrm{FBP}=$ filtered back-projection; IR $=$ iterative reconstruction; $\mathrm{LDCT}=$ low-dose $\mathrm{CT}$; NICU = neurosurgical intensive care unit; SAFIRE = sinogram-affirmed iterative reconstruction; SDCT = standard-dose CT; ULDCT = ultra-low-dose CT

C T by using iterative reconstruction (IR), an alternative to legacy filtered back-projection (FBP), is now ubiquitously available. IR methods loop iteratively through the image reconstruction, reducing noise, with each pass permitting the use of lower levels of ionizing radiation while preserving acceptable image quality. ${ }^{1,2}$ IR methods

Received November 1, 2013; accepted after revision December 5.

From the Department of Neuroradiology, The Mount Sinai Medical Center, New York, New York.

Idoia Corcuera-Solano was supported by a grant from the Spanish foundation: Fundación Alfonso Martín Escudero.

Paper previously presented in part at: 37th Annual Meeting of the European Society of Neuroradiology, September 28-October 1, 2013; Frankfurt, Germany.

Please address correspondence to Lawrence N. Tanenbaum, MD, Mount Sinai Medical Center Department of Radiology, B1234, One Gustave L. Levy Place New York, NY 10029; e-mail: nuromri@gmail.com

- Indicates open access to non-subscribers at www.ajnr.org

http://dx.doi.org/10.3174/ajnr.A3861 have been successfully applied in cardiovascular, ${ }^{3,4}$ thoracic, ${ }^{5-8}$ abdominal,,${ }^{9,10}$ and head CT applications. ${ }^{11-14}$ This study used a commercially available advanced IR technique, sinogram-affirmed iterative reconstruction. Sinogram-Affirmed Iterative Reconstruction (SAFIRE) is a raw data and image domain-based ${ }^{15}$ noise-modeling technique with 5 user-selectable strength levels. Previous work established the feasibility of SAFIRE for the study of body regions ${ }^{15-20}$ and more recently in head $\mathrm{CT}^{21}$ applications.

Patients in the neurosurgical intensive care unit (NICU) typically undergo multiple head CT examinations, resulting in high cumulative radiation exposures. In this study, we evaluated radiation dose and image quality of head CTs obtained with a NICUdesignated ultra-low-dose (ULDCT) protocol (120 dose-modulated effective milliampere-second), with a dose index volume $\left(\mathrm{CTDI}_{\mathrm{vol}}\right)$ approximately $80 \%$ below the recommended reference level in the current American College of Radiology guidelines. ${ }^{22}$ We compared these studies with our standard-of-care low-dose 


\begin{tabular}{|c|c|c|c|}
\hline & \multirow{2}{*}{$\begin{array}{c}\text { FBP } \\
\text { SDCT }\end{array}$} & \multicolumn{2}{|c|}{ SAFIRE } \\
\hline & & LDCT & ULDCT \\
\hline Scanner type & Siemens Somaton Sensation 64 scanner & Siemens Definition AS +128 & Siemens Definition AS +128 \\
\hline $\mathrm{kV}$ (peak) & 120 & 100 & 100 \\
\hline $\mathrm{mAs}$ & 350 & 290 & 120 \\
\hline Section thickness & 4.8 & 5 & 5 \\
\hline Reconstruction kernel & 340 & $J 40 f$ & $J 40 f$ \\
\hline Pitch & 0.6 & 0.6 & 0.6 \\
\hline Rotation time (sec) & 0.5 & 0.5 & 0.5 \\
\hline
\end{tabular}

an-plane, through-plane, and lens protective dose-modulation (X-CARE; Siemens) were used.

(LDCT) IR protocol (290 dose-modulated effective mAs), also SAFIRE-based, and with our legacy standard-dose (SDCT) FBP protocol (350 fixed milliampere-second). To our knowledge, the use of SAFIRE to support ultra-low-dose head CT for the repeated, approximately daily surveillance examinations in the vulnerable NICU population has not been reported.

\section{MATERIALS AND METHODS \\ Patients}

The institutional review board approved this retrospective singlecenter study, with a waiver of informed consent. Our institutional radiology data base was queried to identify NICU patients who underwent head CT by using ULDCT from December 2012 through February 2013. Sixty head CT examinations of 28 consecutive NICU patients were reviewed. We selected patients who underwent head CT using ULDCT, having at least 1 LDCT and/or SDCT for comparison. Six patients were excluded from the image analysis, 4 due to the lack of LDCT or SDCT available for comparison and 2 due to metallic artifacts caused by surgical material. Fifty-four head CT examinations, ULDCT $(n=22)$, LDCT $(n=$ 12 ), and SDCT $(n=20)$, from 22 patients were included for analysis.

\section{CT Protocol Selection}

Scanner selection was based on availability. If the patient was scanned on the non-IR-equipped machine, a FBP-based SDCT was performed. If the IR-equipped scanner was available, our standard clinical LDCT was used for the initial evaluation. If the patient was seen subsequently, the surveillance ULDCT protocol was used whenever the IR-capable scanner was available. Because NICU patients underwent multiple examinations, many had SDCT and LDCT along with ULDCT examinations on sequential days, affording the opportunity to assess quality and dose across the examination types in individual patients.

\section{CT Data Acquisition}

SDCT was performed with FBP on a variety of clinical scanners unequipped with IR capability, most on a Somatom Sensation 64 scanner (Siemens, Erlangen, Germany). LDCT and ULDCT were performed on a Definition AS+128 scanner (Siemens). CT data parameters are summarized in Table 1.

\section{Radiation Dose Assessment}

The CT dose index volume in milligrays and the dose-length product were extracted from the scan-dose page. Effective dose in millisieverts was estimated by multiplying the dose-length product with a constant region-specific conversion coefficient of
$0.0023 \mathrm{mSv} /(\mathrm{mGy} \times \mathrm{cm}) .{ }^{23}$ Minimum, mean, and maximum doses of $\mathrm{CTDI}_{\mathrm{vol}}$, dose-length product and effective dose in the 3 subgroups were compared.

The difference in doses was calculated by subtracting the mean CTDI $_{\mathrm{vol}}$ used in examinations with ULDCT from the mean CTDI $_{\mathrm{vol}}$ of SDCT. The mean CTDI ${ }_{\mathrm{vol}}$ obtained from LDCT was subtracted from the SDCT values; and ULDCT, from LDCT. A percentage difference was also calculated. The same analysis was performed with the dose-length product and effective dose.

\section{Image Quality Analysis}

Quantitative Analysis. A board-certified radiologist with 1 year of neuroradiology experience (A.N.), not involved in the subjective analysis, performed the quantitative measurements. ROIs were placed in the white matter by using an analysis and viewing workstation (Advantage Workstation 4.6; GE Healthcare, Milwaukee, Wisconsin). We drew identical circular $10-\mathrm{mm}^{2}$ ROIs in the corona radiata, avoiding volume-averaging with sulci, cisterns, and gray matter. The following quantitative parameters were acquired for each set of images:

1) Signal (S), defined as the mean CT attenuation values in Hounsfield units.

2) Image noise (IN), defined as the SD of CT attenuation values.

3) $\mathrm{SNR}=($ Mean WM Region of Interest $) /($ SD WM Region of Interest) or (S)/(IN).

Qualitative Analysis. Two board-certified neuroradiologists, with 10 and $\geq 25$ years of experience (A.H.D., L.N.T.) independently analyzed the 3 sets of images. Readers were blinded to reconstruction methods and radiation dose. Readers were asked to evaluate image granularity, defined as the overall graininess or mottle; gray matter-white matter differentiation, defined as the perceived contrast between the gray and white matter; and overall image quality of each CT image on a 5-point scale (Table 2).

Direct pair-wise blinded comparison was performed within each of the ULDCT-SDCT, LDCT-SDCT, and ULDCT-LDCT image sets for each patient. Window and level settings were standardized for initial review, but each reader was also allowed to vary the settings. Image quality scores were averaged across both readers for analysis and are presented as mean value and SD.

\section{Statistical Analysis}

Statistical analyses were performed by using the commercially available IBM Statistical Package for the Social Sciences software (Version 20; IBM, Armonk, New York). Radiation variables were 
Table 2: Grading system for the qualitative assessment

\begin{tabular}{clll}
\hline Score & \multicolumn{1}{c}{ Granularity } & GM-WM Differentiation & \multicolumn{1}{c}{ Overall Quality } \\
\hline 1 & Much more than expected & Much worse than expected & Much worse than expected \\
2 & More than expected & Worse than expected & Worse than expected \\
3 & Normal, as expected & Normal, as expected & Equal, as expected \\
4 & Less than expected & Better than expected & Better than expected \\
5 & Much less than expected & Much better than expected & Much better than expected \\
\hline
\end{tabular}

Table 3: Radiation dose for the ULDCT, LDCT, and SDCT

\begin{tabular}{lrcc}
\hline & $\begin{array}{c}\text { ULDCT } \\
(\boldsymbol{n}=\mathbf{2 2})\end{array}$ & $\begin{array}{c}\text { LDCT } \\
(\boldsymbol{n}=12)\end{array}$ & $\begin{array}{c}\text { SDCT } \\
(\boldsymbol{n}=\mathbf{2 0})\end{array}$ \\
\hline CTDI $_{\text {vol }}(\mathrm{mGy})$ & & & \\
Max & 20.46 & 54.71 & 60.69 \\
Min & 11.77 & 26.79 & 33.80 \\
Mean & 15.55 & 36.47 & 48.38 \\
DLP (mGy·cm) & & & \\
Max & 375.00 & 958.00 & 1128.20 \\
Min & 188.00 & 471.00 & 574.65 \\
Mean & 273.39 & 668.58 & 843.30 \\
ED (mSv) & & & \\
Max & 0.86 & 2.20 & 2.59 \\
Min & 0.43 & 1.08 & 1.32 \\
Mean & 0.62 & 1.53 & 1.93 \\
\hline
\end{tabular}

Note:-Max indicates maximum; Min, minimum; DLP, dose-length product; ED, effective dose.

compared by using the Student $t$ test for paired samples. A nonparametric paired Wilcoxon test was used to perform inter-CT protocol comparisons between the image quality parameters. $\mathrm{Nu}-$ meric data were expressed as mean \pm SD. Image quality scores, signal, image noise, and SNR for each set of CT images were analyzed. Difference was considered statistically significant at $P<$ .05 . Interobserver agreement for assessment of image quality was quantified by weighted $\kappa$ statistics. $^{24}$

\section{RESULTS}

\section{Patient Groups}

Scans of 22 patients (12 men, 10 women; mean age, $59 \pm 0.81$ years; range, $21-87$ years) were analyzed. Ten patients had scans with ULDCT, LDCT, and SDCT techniques; 10 had scans with ULDCT and SDCT; and 2, with ULDCT and LDCT. The mean time interval between initial imaging with SDCT and ULDCT follow-up was 2.3 days; between SDCT and LDCT, it was 6.5 days; and between LDCT and ULDCT, 8.3 days.

All patients underwent CT examinations only for clinical assessment or follow-up. The reasons for scanning included ischemic stroke $(n=7)$, external ventricular drain placement $(n=5)$, hemorrhagic stroke $(n=4)$, subdural hematoma $(n=1)$, aneurysmal subarachnoid hemorrhage $(n=3)$, deep brain stimulation placement $(n=1)$, and brain tumor $(n=1)$.

\section{Radiation Exposure}

Differences in mean CTDI $_{\text {vol }}$, dose-length product, and effective dose between groups were statistically significant (all, $P<.01$ ).

A $68 \%$ reduction of the mean CTDI $_{\text {vol }}(15.55 \mathrm{mGy})$ was found with ULDCT compared with $48.38 \mathrm{mGy}$ in the SDCT group. A $67 \%$ reduction of the mean dose-length product $(273.39 \mathrm{mGy} \cdot$ $\mathrm{cm})$ and $68 \%$ of the mean effective dose $(0.62 \mathrm{mSv})$ were found when using ULDCT compared with the $843.30 \mathrm{mGy} \cdot \mathrm{cm}$ and 1.93 $\mathrm{mSv}$ of SDCT, respectively.
When we used LDCT compared with the SDCT, a 24\% reduction of the mean $\mathrm{CTDI}_{\mathrm{vol}}$ and a $21 \%$ reduction of the mean doselength product and the mean effective dose was found.

A $57 \%$ reduction of the mean $\mathrm{CTDI}_{\mathrm{vol}}$ and a $59 \%$ reduction of the mean dose-length product and the mean effective dose were found when using ULDCT compared with the LDCT scans (Tables 3 and 4).

\section{Quantitative Analysis}

ULDCT was comparable with SDCT with respect to SNR, albeit with a significant increase of image noise $(P=.001)$. The signal was significantly higher in ULDCT scans $(P=.007)$. LDCT was comparable in terms of signal, image noise, and SNR with SDCT. ULDCT, performed with a $58 \%$ lower tube current setting than LDCT, had an expected relatively higher image noise $(P=.003)$ and lower SNR $(P=.001)$. No significant differences were found in the signal (Table 5 and Fig 1).

\section{Qualitative Analysis}

The interobserver agreement in the assessment of image quality parameters was good $(\kappa=0.64)$. No significant difference in overall quality was found between ULDCT and SDCT. The scores for ULDCT were significantly higher than those for SDCT for GM-WM differentiation $(P=.004)$, while ULDCT was associated with significantly more granularity $(P=.023)$. LDCT was associated with significantly less granularity $(P=$ $.032)$ and better overall quality $(P=.022)$ compared with SDCT and resulted in a significant improvement of GM-WM differentiation $(P=.034)$. Scores of the ULDCT and LDCT protocols for GM-WM differentiation were comparable, while LDCT was associated with significantly less granularity and better overall quality ( $P=.003$ ) (Table 6 and Fig 2$)$. Image examples are provided in Figs 3 and 4.

\section{DISCUSSION}

Concern over the potential dangers of high radiation exposures from CT scans has been growing in recent years. ${ }^{25}$ We were particularly interested in reducing the cumulative exposure for the vulnerable NICU patient population-subjects who typically undergo multiple, often daily, head CT examinations for days to weeks; thus, we adopted a specific ultra-low-dose clinical protocol for these surveillance studies.

IR techniques reduce image noise, allowing the use of lower CT doses while preserving image quality. ${ }^{26}$ Recently, a secondgeneration variation of iterative reconstruction for CT, SAFIRE, has become available for clinical use. To the best of our knowledge, this is the first study to evaluate the efficacy and adequacy of an extremely low-dose, special-purpose head CT protocol $\left(\mathrm{CTDI}_{\mathrm{vol}}, 15.55 \mathrm{mGy}\right.$ ) by using SAFIRE, against standard-of-care examinations by using IR and FBP in the same patient. We com- 
pared 3 clinical CT protocols: 350 fixed milliampere-second SDCT with FBP, 290 effective milliampere-second LDCT, and 120 effective milliampere-second ULDCT (both LDCT and SDCT reconstructed with SAFIRE), performed on a consecutive group of NICU patients.

When the ULDCT studies were compared with SDCT, image overall quality was equivalent despite a $66 \%$ relative dose reduction. At a relative dose reduction of $17 \%$, SAFIRE-based LDCT scored significantly higher for subjective image quality, with no differences in noise compared with SDCT images reconstructed with FBP. When the 2 SAFIRE techniques were compared, ULDCT had significantly higher image noise and lower image quality than LDCT. This finding was consistent with expectations that image quality will be proportional to the dose level used when all other parameters are fixed. Our findings are concordant with previous studies using SAFIRE-based CT. Kalra et $\mathrm{al}^{18}$ compared the use of abdominal CT reconstructed with SAFIRE with CT reconstructed with FBP and demonstrated a 50\% reduced dose and, in some patients, a $75 \%$ reduced dose when using SAFIRE without a loss of diagnostic value. Similarly, Winklehner et al ${ }^{19}$ showed the potential to reduce the radiation dose by $>50 \%$ in body CTA studies by using SAFIRE without a deterioration in image quality. In a chest CT study, Pontana et $\mathrm{al}^{16}$ demonstrated a $50 \%$ lower dose while image quality was preserved. SAFIRE has also been recently applied in the study of cervical spine CT, providing better image quality for intervertebral disks, the neural foramina, and ligaments compared with FBP, while reducing the radiation dose by approximately $40 \% .^{20}$ Korn et $\mathrm{al}^{21}$ recently published the first study of the use of SAFIRE in head CT and demonstrated that at $20 \%$ dose reduction, image quality was better with SAFIRE than with standard-dose FBP.

In the literature, there is a wide variation of dose-reduction results by using SAFIRE, ${ }^{15-21}$ which makes it difficult to compare results. Differences might be partially explained by the inconsistency

Note:-DLP indicates dose-length product; ED, effective dose

\begin{tabular}{lc}
$10 \%$ & $62 \%$ \\
$20 \%$ & $56 \%$ \\
$24 \%$ & $57 \%$ \\
& \\
$15 \%$ & $61 \%$ \\
$18 \%$ & $61 \%$ \\
$21 \%$ & $59 \%$ \\
& \\
$15 \%$ & $61 \%$ \\
$17 \%$ & $60 \%$ \\
$21 \%$ & $59 \%$ \\
\hline
\end{tabular}

Table 5: Quantitative analysis: signal, image noise, and SNR in ULDCT, LDCT, and SDCT ${ }^{\text {a }}$

\begin{tabular}{|c|c|c|c|c|c|c|c|c|c|}
\hline & ULDCT & SDCT & $P$ & LDCT & SDCT & $\boldsymbol{P}$ & ULDCT & LDCT & $P$ \\
\hline $\mathrm{S}(\mathrm{HU})$ & $32.94 \pm 4.16$ & $29.59 \pm 4.97$ & .007 & $31.67 \pm 4.27$ & $29.59 \pm 4.97$ & .359 & $32.94 \pm 4.16$ & $31.67 \pm 4.27$ & .320 \\
\hline IN (HU) & $5.05 \pm 0.92$ & $4.40 \pm 0.89$ & .001 & $3.81 \pm 0.40$ & $4.40 \pm 0.89$ & .154 & $5.05 \pm 0.92$ & $3.81 \pm 0.40$ & .003 \\
\hline SNR & $6.71 \pm 1.38$ & $6.91 \pm 1.45$ & .251 & $8.35 \pm 1.2$ & $6.91 \pm 1.45$ & .164 & $6.71 \pm 1.38$ & $8.35 \pm 1.2$ & .001 \\
\hline
\end{tabular}

Note:-S indicates signal; IN, image noise.

${ }^{a}$ Data are presented as mean and SD.
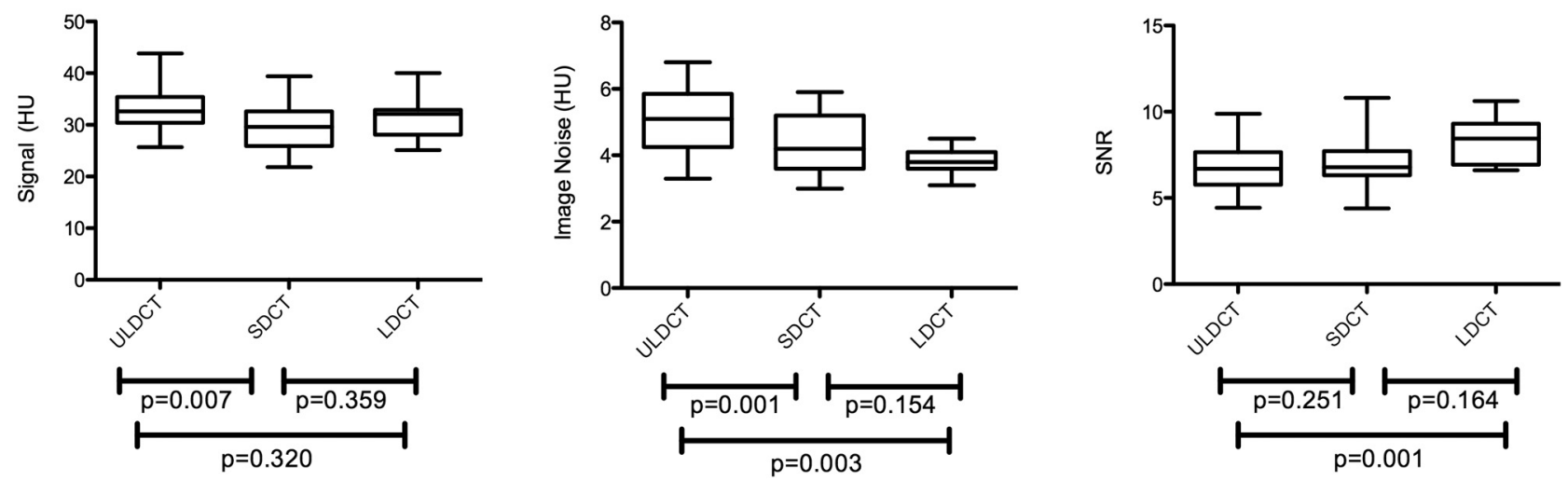

FIG 1. Boxplot diagrams. Signal, image noise, and SNR for SDCT, LDCT, and ULDCT are depicted. The line across the middle of the box identifies the mean sample value; boxes extend from the 25 th to the 75 th quartile, and whiskers, down to the minimum (min) and maximum (max) values.

Table 6: Qualitative analysis of granularity: GM-WM differentiation and overall quality in ULDCT, LDCT, and SDCT ${ }^{\mathrm{a}}$

\begin{tabular}{|c|c|c|c|c|c|c|c|c|c|}
\hline & ULDCT & SDCT & $P$ & LDCT & SDCT & $P$ & ULDCT & LDCT & $P$ \\
\hline Granularity & $2.82 \pm 0.47$ & $3.38 \pm 0.58$ & .023 & $4 \pm 0.42$ & $3.38 \pm 0.58$ & .032 & $2.82 \pm 0.47$ & $4 \pm 0.42$ & .003 \\
\hline GM-WM diff & $3.50 \pm 0.67$ & $2.90 \pm 0.59$ & .004 & $3.79 \pm 0.49$ & $2.90 \pm 0.59$ & .034 & $3.50 \pm 0.67$ & $3.79 \pm 0.49$ & 277 \\
\hline Overall quality & $3.25 \pm 0.45$ & $3.25 \pm 0.52$ & .908 & $4.08 \pm 0.51$ & $3.25 \pm 0.52$ & .022 & $3.25 \pm 0.45$ & $4.08 \pm 0.51$ & .003 \\
\hline
\end{tabular}

Note:-GM-WM diff indicates gray matter-white matter differentiation.

${ }^{a}$ Data are presented as mean and SD. 
granularity

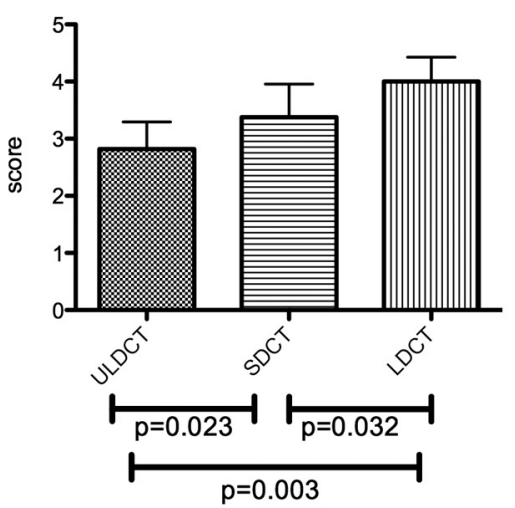

G-W matter differentiation

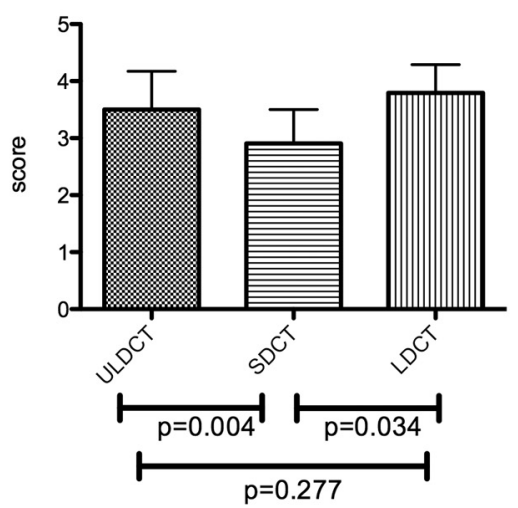

Overall Quality

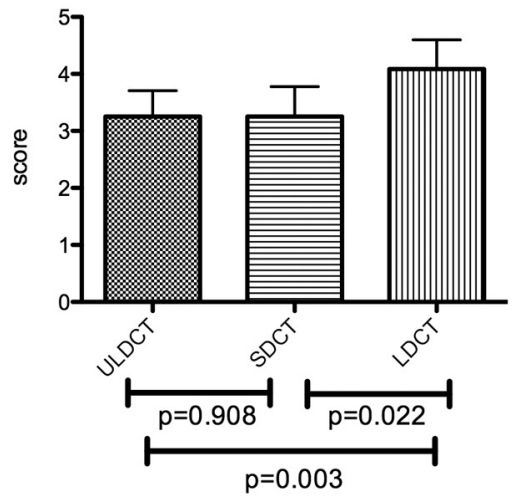

FIG 2. Qualitative analysis of granularity, GM-WM differentiation, and overall quality in ULDCT, LDCT, and SDCT. Data are presented as mean and SD.

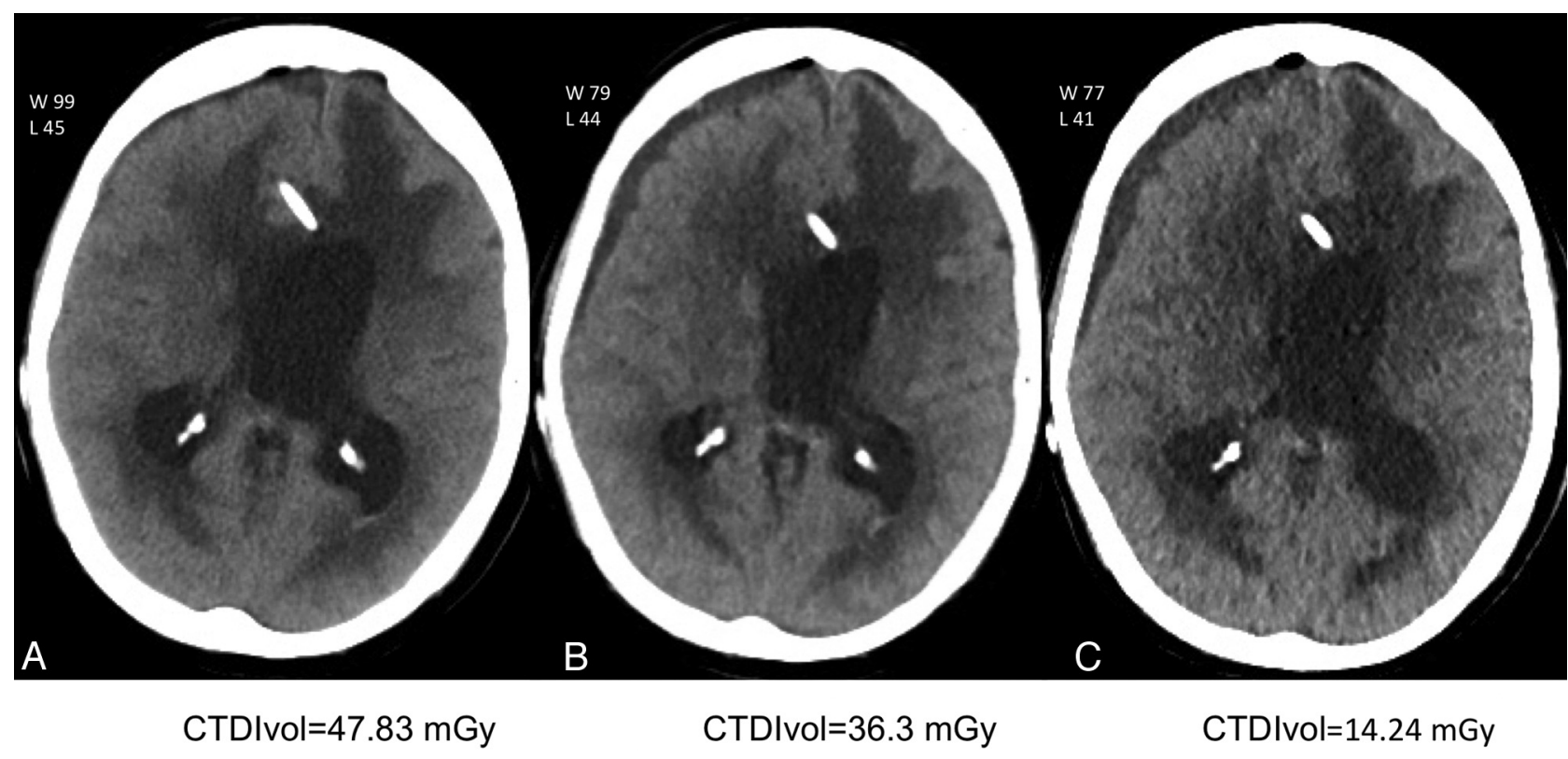

FIG 3. Comparison of SDCT-reconstructed FBP, LDCT, and ULDCT with SAFIRE obtained during 1 week in a 66-year-old NICU patient. Initial scan performed as an FBP SDCT (A) shows the reference image quality. Follow-up SAFIRE LDCT 24 hours later (B) and ULDCT SAFIRE (C) performed 72 hours after the initial examination, at approximately the same level as $A$.

in the method used to calculate dose reductions ${ }^{26}$ and by the use of different scanners in those studies.

There are several aspects of our study that complement the published material to date. The ULDCT, LDCT, and SDCT scans were all obtained in the same patient cohort so that a more accurate intrapatient comparison could be performed. Additionally, we focused exclusively on the acceptability of a special-purpose, dedicated protocol for NICU patients - a vulnerable population subset in whom the frequent, repeated use of the head CT is of particular concern. Our approach used a significantly dose-reduced SAFIRE protocol, 58\% lower than our routine clinical IR protocol and 53\% lower than the low-dose protocol used in the Korn et al study. ${ }^{21}$ While they reported a CTDI ${ }_{\mathrm{vol}}$ of $47.8 \mathrm{mGy}$ in their low-dose CT protocol, ${ }^{21}$ similar to the dose in our clinical standard LDCT, our ULDCT had a mean $\mathrm{CTDI}_{\mathrm{vol}}$ of $15.55 \mathrm{mGy}$, representing a $67 \%$ reduction in comparison, and it was 79\% lower than the 75-mGy limit recommended by the American College of Radiology guidelines. ${ }^{22}$
An optimal CT protocol uses the lowest dose and provides the appropriate image quality for the clinical circumstances for which it is intended. Head CT is a critical tool for the surveillance of the NICU population, typically used serially to monitor and guide treatment. We presumed that a protocol with drastically reduced doses would be adequate for these surveillance examinations used primarily to follow known abnormalities such as hematoma size, catheter position, and ventricular caliber and that they would be clinically acceptable at a somewhat reduced level of quality and low contrast detectability than a routine CT. This retrospective study confirmed that this ULDCT approach provided acceptable quality for these surveillance examinations in the NICU population.

An important question concerns the level of dose reduction obtained before diagnostic accuracy is impaired. An optimal combination of SAFIRE and dose reduction has not yet been established. This study demonstrated the ability of reducing the 

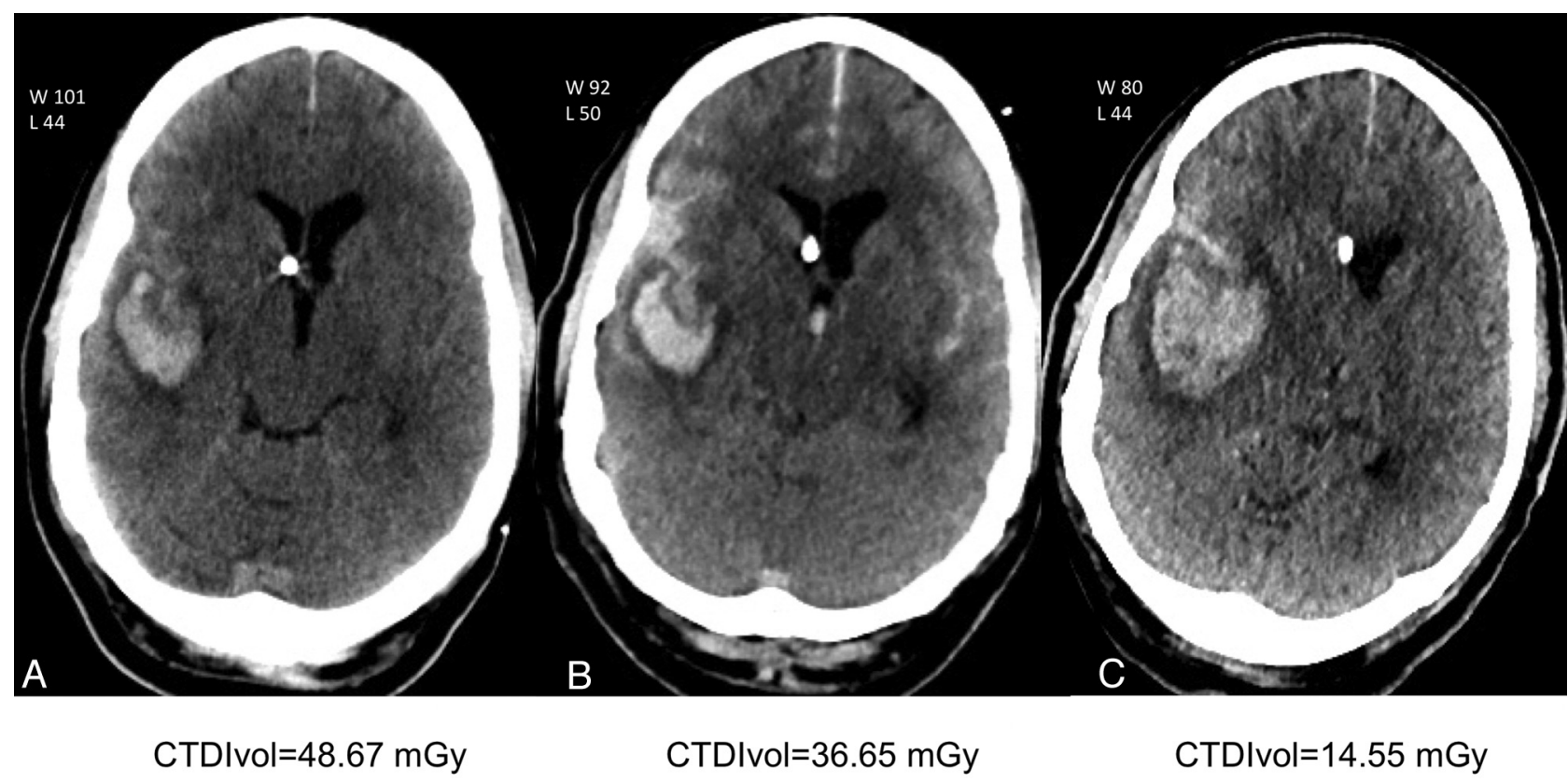

CTDlvol=36.65 mGy

CTDIvol=14.55 $\mathrm{mGy}$

FIG 4. Comparison of FBP SDCT, SAFIRE LDCT, and ULDCT obtained over the course of 1 week in a 55-year-old NICU patient after Onyx (ev3, Irvine, California) embolization of a right parietotemporal AVM. SDCT image $(A)$ obtained shortly after treatment shows the reference image quality. Follow-up LDCT 5 days later $(B)$ and ULDCT SAFIRE $(C)$ images obtained 7 days after the initial examination at approximately the same level as $A$. Note that the right parietotemporal hemorrhage is well-seen with both the LDCT and ULDCT studies.

dose up to $69 \%$ and maintaining diagnostic quality for this special purpose.

We acknowledge several study limitations. First, the retrospective design and the small sample size, with only 12 cases with dedicated ULDCT and LDCT for comparison, requires a confirmation of our findings in a prospective trial and a larger population. Second, while reviewers were blinded to protocol and reconstruction technique, blinding was not perfect because the varied use of scanner and reconstruction methods left subtle but recognizable differences in the appearance of the datasets. Third, different scanners were used for the SAFIRE and FBP groups; hence, we are unable to exclude the possible influence of different detector efficiency, image thickness, and reconstruction kernels on image quality and radiation dose. Finally, these results apply only to the specified protocols and CT scanners and will not translate exactly to other protocols and scanners, though the fundamentals with respect to a radiation-vulnerable population should apply in a vendor and IR agnostic fashion.

\section{CONCLUSIONS}

This study suggests that ULDCT with SAFIRE is a viable technique to significantly decrease radiation exposure while preserving image quality for the surveillance of the NICU population who are undergoing repeated head scanning. ULDCT could replace conventional CT for follow-up evaluations of the brain whenever serial examinations are performed or anticipated.

\section{ACKNOWLEDGMENTS}

We acknowledge John Doucette, $\mathrm{PhD}$, for his statistical assistance during the development of this manuscript and Mark Smith, LRT (R), for his assistance at the CT scanners.
Disclosures: Idoia Corcuera-Solano-RELATED: supported by a grant from the Spanish foundation: Fundación Alfonso Martín Escudero. Lawrence N. Tanenbaum-RELATED: a speaker for Siemens.

\section{REFERENCES}

1. Nuyts J, De Man B, Dupont P, et al. Iterative reconstruction for helical CT: a simulation study. Phys Med Biol 1998;43:729-37

2. Thibault JB, Sauer KD, Bouman CA, et al. A three-dimensional statistical approach to improved image quality for multislice helical CT. Med Phys 2007;34:4526-44

3. Tumur $\mathrm{O}$, Soon $\mathrm{K}$, Brown $\mathrm{F}$, et al. New scanning technique using Adaptive Statistical Iterative Reconstruction (ASIR) significantly reduced the radiation dose of cardiac CT. J Med Imaging Radiat Oncol 2013;57:292-96

4. Gosling O, Loader R, Venables $\mathrm{P}$, et al. A comparison of radiation doses between state-of-the-art multislice CT coronary angiography with iterative reconstruction, multislice CT coronary angiography with standard filtered back-projection and invasive diagnostic coronary angiography. Heart 2010;96:922-26

5. Leipsic J, Nguyen G, Brown J, et al. A prospective evaluation of dose reduction and image quality in chest CT using adaptive statistical iterative reconstruction. $A J R A m$ J Roentgenol 2010;195:1095-99

6. Pontana F, Pagniez J, Flohr T, et al. Chest computed tomography using iterative reconstruction vs filtered back projection. Part 1. Evaluation of image noise reduction in 32 patients. Eur Radiol 2011;21:627-35

7. Pontana F, Duhamel A, Pagniez J, et al. Chest computed tomography using iterative reconstruction vs filtered back projection. Part 2. Image quality of low-dose CT examinations in 80 patients. Eur Radiol 2011;21:636-43

8. $\mathrm{Xu} \mathrm{Y}, \mathrm{He} \mathrm{W}$, Chen $\mathrm{H}$, et al. Impact of the adaptive statistical iterative reconstruction technique on image quality in ultra-low-dose CT. Clin Radiol 2013;68:902-08

9. Singh S, Kalra MK, Hsieh J, et al. Abdominal CT: comparison of adaptive statistical iterative and filtered back projection reconstruction techniques. Radiology 2010;257:373-83 
10. Sagara Y, Hara AK, Pavlicek W, et al. Abdominal CT: comparison of low-dose $\mathrm{CT}$ with adaptive statistical iterative reconstruction and routine-dose $\mathrm{CT}$ with filtered back projection in $\mathbf{5 3}$ patients. AJR Am J Roentgenol 2010;195:713-19

11. Kilic K, Erbas G, Guryildirim M, et al. Lowering the dose in head CT using adaptive statistical iterative reconstruction. AJNR Am J Neuroradiol 2011;32:1578-82

12. Korn A, Fenchel M, Bender B, et al. Iterative reconstruction in head $\mathrm{CT}$ : image quality of routine and low-dose protocols in comparison with standard filtered back-projection. AJNR Am J Neuroradiol 2012;33:218-24

13. Rapalino O, Kamalian S, Kamalian S, et al. Cranial CT with adaptive statistical iterative reconstruction: improved image quality with concomitant radiation dose reduction. AJNR Am J Neuroradiol 2012;33:609-15

14. Vorona GA, Zuccoli G, Sutcavage T, et al. The use of adaptive statistical iterative reconstruction in pediatric head CT: a feasibility study. AJNR Am J Neuroradiol 2013;34:205-11

15. Moscariello A, Takx RA, Schoepf UJ, et al. Coronary CT angiography: image quality, diagnostic accuracy, and potential for radiation dose reduction using a novel iterative image reconstruction technique-comparison with traditional filtered back projection. Eur Radiol 2011;21:2130-38

16. Pontana F, Pagniez J, Duhamel A, et al. Reduced-dose low-voltage chest CT angiography with sinogram-affirmed iterative reconstruction versus standard-dose filtered back projection. Radiology 2013;267:609-18

17. Baumueller S, Winklehner A, Karlo C, et al. Low-dose CT of the lung: potential value of iterative reconstructions. Eur Radiol 2012;22:2597-606
18. Kalra MK, Woisetschlager M, Dahlstrom N, et al. Radiation dose reduction with sinogram affirmed iterative reconstruction technique for abdominal computed tomography. J Comput Assist Tomogr 2012;36:339-46

19. Winklehner A, Karlo C, Puippe G, et al. Raw data-based iterative reconstruction in body CTA: evaluation of radiation dose saving potential. Eur Radiol 2011;21:2521-26

20. Becce F, Ben Salah Y, Verdun FR, et al. Computed tomography of the cervical spine: comparison of image quality between a standard-dose and a low-dose protocol using filtered backprojection and iterative reconstruction. Skeletal Radiol 2013;42: 937-45

21. Korn A, Bender B, Fenchel M, et al. Sinogram affirmed iterative reconstruction in head CT: improvement of objective and subjective image quality with concomitant radiation dose reduction. Eur Radiol 2013;82:1431-35

22. McCollough C, Branham T, Herlihy V, et al. Diagnostic reference levels from the ACR CT Accreditation Program. J Am Coll Radiol 2011;8:795-803

23. Bongartz G, Golding SJ, Jurik AG, et al. European Guidelines on Quality Criteria for Computed Tomography. 1999. http://www. drs.dk/guidelines/ct/quality. Accessed October 1, 2013

24. Landis JR, Koch GG. The measurement of observer agreement for categorical data. Biometrics 1977;33:159-74

25. Brenner DJ. Slowing the increase in the population dose resulting from CT scans. Radiat Res 2010;174:809-15

26. Willemink MJ, Leiner T, de Jong PA, et al. Iterative reconstruction techniques for computed tomography. Part 2. Initial results in dose reduction and image quality. Eur Radiol 2013;23:1632-42 